\title{
Uma Análise Exploratória dos Programas de Controle da Tuberculose da Bahia e Goiás à Luz da Teoria dos Custos de Transação'
}

An Exploratory Analysis of the Tuberculosis Control Program
of the States of Bahia and Goiás from a Transaction Cost Theory Perspective

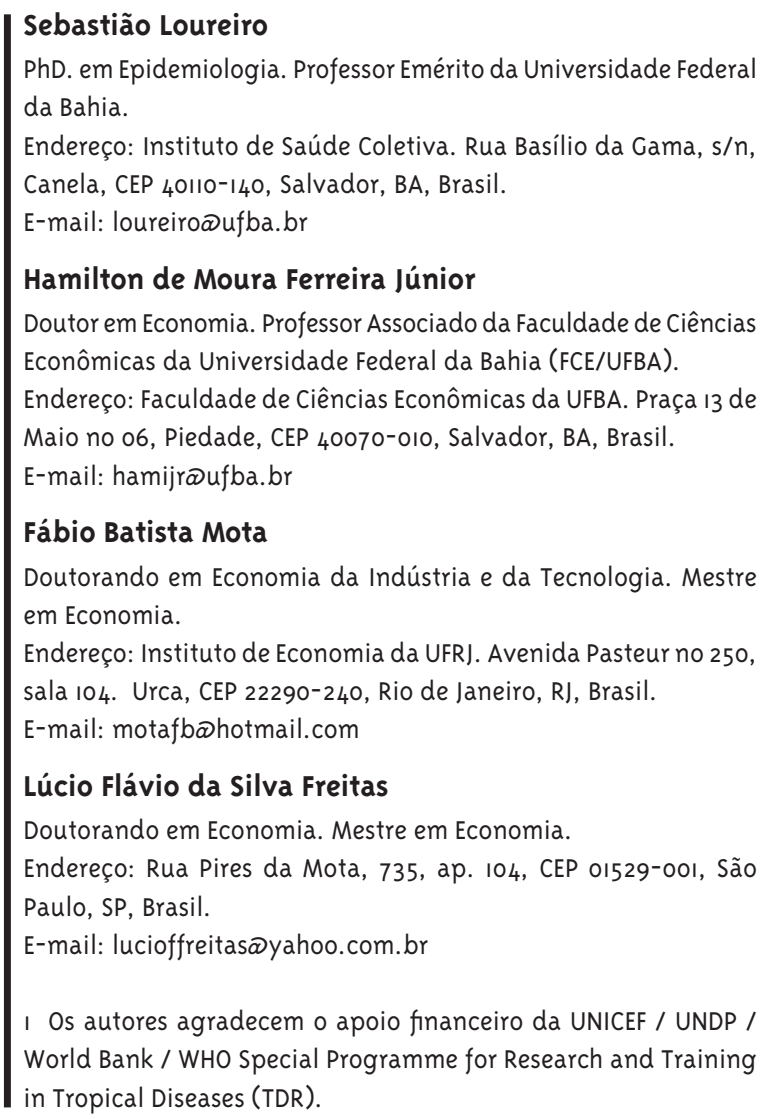

\section{Resumo}

Em caráter exploratório, o objetivo deste artigo é analisar o Programa de Controle da Tuberculose (PCTs) dos Estados da Bahia e Goiás e respectivas capitais, Salvador e Goiânia, a partir da Teoria dos Custos de Transação. Para tanto, foi realizado um estudo de caso nos PCTs citados, utilizando-se, junto aos seus gestores, do método de entrevistas aprofundadas semidiretivas. Os resultados sugerem: (a) baixa especificidade em ativos humanos; (b) baixo grau de incerteza - relacionada à flutuação da demanda e à introdução de novas tecnologias (medicamentos); (c) aspectos relacionados à racionalidade limitada (informação incompleta) são pouco relevantes - no que tange à redação do contrato (Programa Nacional de Controle da Tuberculose - PNCT) e ao Sistema de Informação de Agravos de Notificação (SINAN); (d) e alta probabilidade de ocorrência de comportamento de tipo oportunista (risco moral) - devido ao não monitoramento das ações, à ausência de punições em caso de descumprimento das ações pactuadas no PNCT e ao regime de incentivos vigente.

Palavras-chave: Teoria dos Custos de Transação; Arranjo Institucional; Regime de Incentivos; Programa Nacional de Controle da Tuberculose. 


\section{Abstract}

From a Transaction Cost Theory perspective, the paper aims to discuss the Programa de Controle da Tuberculose (PCT - Tuberculosis Control Program) of the States of Bahia and Goiás and their Capital Cities, Salvador and Goiânia. A case study was carried out applying a research questionnaire to the PCT managers. The results indicate: (a) low specificity in human assets; (b) low uncertainty - related to fluctuations in demand and to the introduction of new technologies (drugs); (c) aspects related to bounded rationality (incomplete information) are not important - related to the contract (PNCT) and the SINAN; (d) and high probability of opportunism (moral hazard), due to lack of monitoring of the actions, lack of punishment in case of default of actions agreed upon in the PNCT, and the current incentive regime.

Keywords: Transaction Cost Theory; Institutional Arrangement; Incentives Regime; National Tuberculosis Control Program.

\section{Introdução}

Em linhas gerais, custos de transação são os custos de negociar, redigir ou garantir o cumprimento de um contrato, num ambiente em que os compromissos são intertemporais. Assim, um contrato deve ser entendido como uma "promessa de conduta futura" e a coordenação como o resultado da ação de instituições que possibilitam a manutenção e ajuste de tais promessas ao longo do tempo (Pondé, 1993). Uma boa revisão de literatura pode ser consultada em Bueno (2004).

Como sabido, a Teoria dos Custos de Transação (TCT) - devida à Coase (1937) e, posteriormente, desenvolvida por Williamson (1989) - foi desenhada originalmente para aplicação em ambientes de mercado, sobretudo para a análise de firmas privadas produtoras de bens. Assim, deslocada tanto do ambiente público quanto da oferta de serviços. Com o tempo, o avanço da teoria permitiu a ampliação do campo dos "fenômenos" explicáveis, expandindo-se, tanto em termos teóricos quanto empíricos, para além das fronteiras analíticas que lhe deram origem. De acordo com Macher e Richman (2008), a TCT tem sido aplicada em áreas como ciências políticas, direito, políticas públicas, agricultura e saúde, por exemplo. No que tange mais especificamente à saúde pública, os autores afirmam que:

Public health has witnessed significant growth in the number of empirical TCE applications, with one of the main impetuses for this recent interest from the dramatic organizational changes affecting the health care industry. These changes, many of which are driven by regulatory measures and advances in medical technology, have led to reorganization in almost every phase of the healthcare industry, most notably the delivery of medical services to patients and payment mechanisms by governments and private insurers (Macher e Richman, 2008, p. 35).

Para estudos que, de forma mais geral, discutem a TCT no ambiente público, vide, e.g., Williamson (1999), Arias e Caballero (2003), Brown e Potoski (2003) e Peres (2007). Para trabalhos mais específicos, envolvendo a aplicação da TCT em estudos sobre serviços públicos e/ou privados de saúde, ver, por exemplo, Ashton (1998), Ashton e colaboradores (2004) e Preker e colaboradores (2000). Outros tra- 
balhos nesta direção são apontados, também, por Macher e Richman (2008), que empreenderam uma avaliação da literatura empírica da TCT no campo das ciências sociais.

Em caráter exploratório, o objetivo deste artigo é analisar o arranjo institucional dos Programas de Controle da Tuberculose (PCT) dos Estados da Bahia e Goiás, e respectivas capitais, Salvador e Goiânia. Para tanto, foi realizado um estudo de caso nas coordenações dos PCTs citados. Tal estudo foi realizado à luz da Nova Economia Institucional (NEI) em sua vertente tradicional, a Teoria dos Custos de Transação. Tal teoria, através de seus supostos básicos, fornece uma heurística adequada à investigação tanto de aspectos relacionados ao comportamento dos agentes quanto daqueles que incidem sobre arranjos institucionais. Aqui, são utilizados, também, elementos da chamada Teoria da Agência, arrolada no campo da NEI, mas apenas no que se refere ao problema básico de agência (como alinhar os objetivos do agente ao do principal?).

Na seção seguinte, apresenta-se o método de pesquisa conjuntamente com uma discussão, a partir da TCT, das suposições arroladas na matriz das características da transação (Quadro1), em sua relação com o "contrato", o Programa Nacional de Controle da Tuberculose (PNCT). A elaboração desta matriz - que orientou o desenvolvimento do estudo de caso - foi inspirada, em grande parte, em Ashton (1998) e UFBA (2007). Informações e dados foram obtidos, também, em várias outras fontes: SINAN; IBGE [s.d.]; World Bank (2007); Secretaria Municipal de Saúde de Goiânia (2007); Santos-Filho e Gomes (2007); Brasil (2006); Souza e colaboradores (2005).

Para o propósito deste estudo, a TCT não foi empregada no sentido de identificar qual seria a estrutura de governança mais adequada (mercado, hierarquia ou formas híbridas) para economizar custos de transação (no caso, nos PCTs pesquisados). Isto, porque, a estrutura de governança que rege a assistência ao tuberculoso é, como não poderia deixar de ser, uma variável dependente da forma como está organizado o Sistema Único de Saúde (SUS). Pode ser considerada, deste modo, um dado do ambiente. Tal discussão envolve, assim, uma noção de eficiência na oferta de serviços públicos de saúde, determinada pelo seu aspecto institucional.
Neste estudo, assim como em Peres (2007, p.17), considera-se que "Não existe, a priori, uma estrutura de governança superior às demais". Nesse sentido, uma discussão sobre a melhoria da eficiência da transação (no caso, a 'assistência ao tuberculoso') deve ser pensada a partir do arranjo institucional dado. Ou seja, no sentido da adequação da estrutura de governança existente às características da transação específica (e.g., tal como disposto no PNCT (Brasil, 2004)). Considerando-se a assistência ao tuberculoso integrada no âmbito do SUS, entende-se a eficiência da hierarquia nos seguintes termos: "[...] a hierarquia é uma estrutura eficiente quando é o melhor que indivíduos auto-interessados conseguem fazer sobre um arranjo institucional possível" (Peres, 2007, p. 19).

Os PCTs das secretarias estaduais de saúde (SES) da Bahia e Goiás, e municipais (SMS), de Salvador e Goiânia, foram escolhidos em função dos indicadores epidemiológicos que apresentam, no plano estadual. Segundo dados do Sistema de Informação de Agravos de Notificação (SINAN) - número de casos novos por UF e número de casos notificados por UF -, para o ano de 2006, a Bahia figurava entre os principais Estados de maior incidência da doença, abaixo, apenas, de, respectivamente, São Paulo e Rio de Janeiro. Goiás, por sua vez, estava localizado na $20^{\mathrm{a}}$ posição, portanto, entre aqueles de menor incidência. Na Bahia, no ano de 2006, ainda segundo dados do SINAN, a taxa de incidência de tuberculose (TB) alcançou 44,2 casos por grupos de 100 mil habitantes, quase três vezes superior àquela verificada em Goiás. O percentual de cura chegou a $67 \%$ e o abandono alcançou 6,1\%. No Estado, em 2005, quase metade da população tinha renda inferior a $1 / 2$ salário mínimo, eram 4,8 enfermeiros para cada cem leitos hospitalares, o,9 médicos por mil habitantes e o Programa de Saúde da Família (PSF) cobria 37\% da população (Brasil, 2006). Em Goiás, para 2006, o SINAN registrou 890 casos novos de tuberculose, correspondendo a uma taxa de incidência de 15,8, por grupos de cem mil habitantes. Os indicadores de tratamento mostraram cura de $58 \%$ e abandono de $8,8 \%$. 0 abandono alcançou $13,1 \%$ dos casos em tratamento. 0 percentual da população coberta pelo PSF foi de 47,4\%, no ano de 2005. À época, um quarto dos residentes no Estado possuía renda mensal 
inferior a 1/2 salário mínimo, o número de médicos por mil habitantes equivalia a 1,2 e havia aproximadamente 12 enfermeiros por cem leitos hospitalares (Brasil, 2006).

Dado o caráter exploratório do estudo, não faz parte do objetivo do trabalho discussões mais aprofundadas sobre a problemática que envolve a prestação de serviços públicos para o combate e controle da TB no Brasil. Isto, inclusive, no que se refere à eficiência do fornecimento destes serviços. A discussão ora realizada está circunscrita ao arranjo institucional dos PCTs citados. Logo, não é passível de generalização para o contexto do PNCT ou demais PCTs estaduais e municipais. Não são realizadas, portanto, afirmações a partir dos resultados do estudo. Os achados permitem, apenas, tecer considerações acerca de características dos arranjos institucionais pesquisados, levando-se em conta os supostos teóricos da TCT e a percepção dos gestores dos PCTs analisados. 0 trabalho apresenta, assim, limitações, que, todavia, são admissíveis em um estudo que se pretende exploratório. 0 mérito está, em parte, na tentativa de exploração e introdução de novos marcos analíticos no campo da Saúde Coletiva e da Economia da Saúde no Brasil.

\section{Método}

De modo a proceder à investigação, realizou-se um estudo de caso nas coordenações dos PCTs das Secretarias Estaduais de Saúde da Bahia e Goiás (SES-BA; GO) e Secretarias Municipais de Saúde de Salvador e Goiânia (SMS-SSA; GNA). Uma vez que a TCT é uma teoria eminentemente qualitativa, optou-se pelo método de entrevistas aprofundadas semidiretivas; no qual os entrevistados respondem subjetivamente a um roteiro de entrevistas. Os tópicos discutidos nas entrevistas abrangeram: (a) especificidade de ativos em capital humano; (b) Incerteza - no que tange aos fatores redutores de incerteza, flutuação da demanda (impactos prováveis e novas tecnologias) e de preços; (c) Oportunismo (risco moral); (d) racionalidade limitada (informação incompleta); (e) e aspectos políticos e administrativos. Os respondentes eram os coordenadores e técnicos dos referidos PCTs; aqui chamados de gestores. A pesquisa de campo foi iniciada em dezembro de 2007 e finalizada em fevereiro de 2008. Nesse período, sete gestores aceitaram participar da pesquisa (especificamente: o1 no PCT-SES-BA; o1 no PCT-SMS-SSA; o 4 no PCT-SES-GO; e o1 no PCT-SMS-GNA). Assim como em Ashton (1998), admite-se que a opção pelo método de análise qualitativa prescinde de uma amostra representativa ou estatisticamente significativa. As respostas foram gravadas, transcritas e checadas antes de serem analisadas; não foi utilizado software de análise qualitativa.

No que tange aos aspectos éticos da pesquisa, respeitou-se a Resolução n⿳ํㅜ 196/96 do Conselho Nacional de Saúde. O projeto que deu origem ao presente artigo foi enviado ao Comitê de Ética em Pesquisa do Instituto de Saúde Coletiva da Universidade Federal da Bahia, tendo sido aprovado (Registro CEP: 034-07/CEP-ISC; PARECER № 054o7/CEP-ISC). Foi utilizado, nas entrevistas com os gestores, um 'termo de consentimento livre e esclarecido do participante' (TCLE), assinado em duas vias, ficando uma em poder do entrevistador e a outra com o respondente. Nele, foi garantido aos entrevistados o tratamento dos dados no agregado e com confidencialidade da fonte da informação, de modo que, para preservar o aspecto ético, não serão, aqui, qualificados; ou seja, nenhuma menção a nome, cargo ou função será realizada.

Admitiu-se, por suposto, que, no Brasil, exclusive a frequência, as características da transação, a assistência ao tuberculoso, seguem os níveis descritos no Quadro 1. Tal matriz resume as hipóteses que foram levantadas para nortear o processo de investigação; especialmente o estudo de caso. A dimensão da transação 'frequência', da relação contratual, não foi incluída no estudo de caso, visto que as informações relevantes para a análise não poderiam ser coletadas ao nível dos PCTs pesquisados (e.g., a frequência da relação contratual entre comprador e fornecedores de medicamentos para tuberculose, respectivamente, Governo Federal e Rede Oficial de Laboratórios). Vale destacar que a análise limitar-se-á, tão somente, aos PCTs pesquisados, não pretendendo realizar extrapolações diretas para o contexto geral do PNCT no âmbito do SUS, bem como acerca dos PCTs das demais unidades da federação. Isto, grosso modo, porque o PNCT e o SUS são aparatos institucionais de grandes dimensões. Ambos possuem 


\section{Quadro I - Matriz das características da transação}

\begin{tabular}{|l|c|c|c|c|c|}
\hline \multirow{2}{*}{ Transação } & \multicolumn{3}{|c|}{ Dimensões da transação } & \multicolumn{3}{c|}{ Pressupostos sobre a conduta humana } \\
\cline { 2 - 6 } & Especificidade de Ativos & Incerteza & Frequência & Racionalidade Limitada & Oportunismo \\
\hline Assistência ao tuberculoso & Baixa & Baixa & - & Baixa & Alta \\
\hline
\end{tabular}

alcance nacional, grande diversidade e exercem os seus papéis em um contexto social e sanitário marcado por fortes e persistentes desigualdades. Assim, é reconhecido que os resultados do presente estudo estão circunscritos aos entes subnacionais citados, não sendo - salvo justificativa em contrário - imediatamente aplicáveis ao restante do País.

Desenvolver uma análise empírica a partir da TCT requer, primeiramente, que se defina a 'transação', ou unidade básica de análise, sob a qual se estabelece, em tese, a 'estrutura de governança' mais adequada para economizar tais custos: mercado, formas híbridas ou hierarquia. A escolha do tipo de estrutura de governança mais adequada pode ser considerada, na teoria, uma função da interação entre as 'características da transação': as três 'dimensões da transação' - especificidade de ativos, incerteza e frequência - e os dois 'pressupostos sobre a conduta humana' - racionalidade limitada e oportunismo. Muito simplificadamente, admite-se que há uma tendência à hierarquia, principalmente, quanto maior a especificidade do ativo e a probabilidade de comportamento oportunista entre os agentes de uma relação contratual. Na TCT, entende-se por 'contrato' não apenas as formas explícitas, mas, também, as informais e tácitas. Neste trabalho, o PNCT foi tomado como 'contrato', pois nele se procura, grosso modo, orientar as ações dos agentes envolvidos na transação (ou a sinalização das "regras do jogo"). Trata-se, obviamente, de um recurso analítico, cujo fim é simplificar o ambiente (de relações contratuais) no qual se desenrola a transação, imerso, como está, na lógica de funcionamento do SUS.

Resumidamente, no PNCT estão dispostos os objetivos, metas e componentes do programa. Tem, por objetivo geral, a redução da morbidade, mortalidade e transmissão da tuberculose (TB). Constituem as metas do PNCT: (a) manter a detecção anual de pelo menos $70 \%$ dos casos estimados de TB; (b) tratar corretamente $100 \%$ dos casos de tuberculose diag- nosticados e curar pelo menos $85 \%$ deles; (c) manter o abandono de tratamento em percentuais considerados aceitáveis (5\%); (d) expandir o tratamento supervisionado para $100 \%$ das unidades de saúde dos municípios prioritários, e pelo menos para 80\% dos bacilíferos destes municípios até 2007; (e) manter registro atualizado dos casos diagnosticados e $100 \%$ do resultado de tratamento; (f) aumentar em 100\% o número de sintomáticos respiratórios examinados (2004/2007); (g) disponibilizar teste anti-HIV para $100 \%$ dos adultos com TB. Os componentes do PNCT estão distribuídos em: (a) vigilância epidemiológica; (b) medidas de proteção; (c) integração com atenção básica; (d) ações integradas de educação em saúde, comunicação e mobilização social; (e) capacitação e treinamento; (f) sustentação político-social; (g) e avaliação, acompanhamento e monitoramento das ações. E, por último, os indicadores de acompanhamento do PNCT, distribuídos em indicadores de cobertura, de processo e de resultado (Brasil, 2004).

Ainda que redigido, o PNCT não pode ser enquadrado como um contrato explícito. Dois são os motivos básicos. O primeiro é que, no Brasil, dado o processo de descentralização dos serviços de saúde, cada uma das três esferas de governo - Federal, Estadual e Municipal -, que compõe a transação, dispõe de autonomia e, desse modo, as ações previstas no PNCT podem ser, apenas, pactuadas. Fato que nos leva ao segundo motivo, qual seja, o contrato não especifica um regime de incentivos - que inclui punições aos agentes desalinhados das ações previstas - mas, apenas, objetivos, metas, componentes, distribuição de competências entre as três esferas de governo e como deve ser feito o monitoramento, através dos indicadores de acompanhamento. De acordo com a TCT, uma das implicações mais imediatas da inexistência de um contrato explícito é a maior probabilidade de ocorrência de comportamentos oportunistas por parte dos agentes que compõem a transação. Inobstante, sabe-se que, no 
Brasil, a pactuação entre os entes federados faz parte do regime de direito administrativo, e, como tal, está sujeita às punições previstas em lei em caso de seu descumprimento; em última instância, a interrupção de repasses de recursos financeiros da esfera federal até a municipal.

No presente estudo, define-se a transação como a 'assistência ao tuberculoso', pois é sobre ela que se desenvolve toda uma estrutura de governança dedicada ao combate e controle da tuberculose. A definição de uma unidade básica de análise - a despeito da simplificação que encerra - constitui um recurso analítico interessante, na medida em que permite reduzir a complexidade das relações contratuais que se estabelecem em dado objeto de estudo. Em outras palavras, a unidade básica de análise permite que se estabeleça, no agregado, uma determinada transação, mesmo sabendo-se que coexistem no interior da própria unidade de análise, vários outros tipos de transação, que caracterizam a complexidade das relações contratuais.

No que se refere à primeira dimensão da transação, a especificidade de ativos, pode-se distinguir, ao menos, quatro tipos: 'especificidade de local', 'especificidade dos ativos físicos', 'especificidade dos ativos humanos' e 'ativos dedicados' (Williamson, 1989). Neste trabalho, será investigado, junto aos gestores, apenas a especificidade de ativos em capital humano. Os demais tipos de ativos citados não são diretamente aplicáveis às atividades desenvolvidas no âmbito dos PCTs pesquisados, objeto deste estudo (para maiores detalhes acerca dos tipos de especificidade de ativos, vide Williamson (1989) e Bueno (2004).

A especificidade em ativos humanos (ou em capital humano) "[...] surge como consequência de processos de learning-by-doing ou de transferências de conhecimento entre firmas" (Bueno, 2004, p.383). Em nosso caso, para a realização da transação, a especificidade em ativos humanos pode ser separada, a título de ilustração, em dois tipos básicos, a saber, diretos e indiretos - que são, na verdade, os casos extremos do arranjo institucional pesquisado. 0 primeiro é composto pelos ativos humanos que atuam diretamente com o paciente: Médicos, Enfermeiros e Agentes de Saúde. Os ativos indiretos, por sua vez, são formados pelos gestores e servidores públicos de saúde - ou seja, políticos e burocracia estatal - que atuam nos planos político, administrativo e operacional do PNCT, na esfera federal, e dos PCTS, no plano estadual e municipal. Neste trabalho, admitiu-se, por suposto, que a especificidade é 'baixa' para ambos os ativos humanos, diretos e indiretos. Isto porque o nível de habilidade requerido para executar as funções que lhe competem na transação, a assistência ao tuberculoso, não são, exatamente, específicas, no sentido de que podem ser alocados em outras atividades sem perda de valor produtivo.

Na Nova Economia Institucional, inclusive na sua abordagem mais tradicional, a Teoria dos Custos de Transação, o tratamento da incerteza é uma questão sem consenso (como quase tudo em economia). $\mathrm{Na}$ NEI, a noção de incerteza pode assumir - variando conforme se aproxima ou se afasta do mainstream do pensamento econômico (teoria neoclássica) - as formas 'procedural ou substantiva', 'fraca ou forte' e 'ambiguidade ou fundamental' (Dequech, 2006). Segundo ainda Dequech (2006), incerteza fundamental é caracterizada pela possibilidade de criatividade e mudança estrutural não predeterminada e admite que eventos futuros não são passíveis de determinação ou conhecimento ex ante. No presente estudo, admitiu-se a incerteza de tipo fundamental (relacionada ao desconhecimento do futuro). Tal entendimento da incerteza é, aparentemente, próximo da noção adotada por Ashton (1998), i.e., no que tange ao desconhecimento do futuro.

No caso da assistência ao tuberculoso, poderíamos supor que flutuações na demanda pelo serviço não são importantes, ou que a incerteza estaria contida em intervalos pouco relevantes. Isto porque a tuberculose é, no Brasil, uma doença endêmica. Significa dizer que é baixa a probabilidade de ocorrência de grandes oscilações na procura. Por exemplo, no Brasil, o cálculo que define o incremento anual na oferta de medicamentos, pelo Governo Federal, é realizado com base no número de casos de tuberculose observado no ano anterior, acrescido de $10 \%$ (UFBA, 2007).

A flutuação da demanda devida à introdução de novas tecnologias (no caso, novos medicamentos) deve ser considerada, também, não relevante, levando em conta que o único fornecedor de medicamentos para tuberculose é o Governo Federal. 
Assim, dado o monopólio legal da oferta, um novo medicamento apenas chega ao mercado se introduzido pela autoridade do Estado. Também não deve ser considerada relevante a flutuação nos preços dos insumos para fabricação de medicamentos para tuberculose. Primeiro, porque não é o Governo Federal que adquire os insumos, mas sim a rede de laboratórios oficiais (UFBA, 2007). Segundo, porque o monopólio da oferta de medicamentos confere ao Governo Federal um elevado poder de barganha diante da rede de laboratórios oficiais, o que, em tese, resultaria em vantagens de preço para o primeiro.

Portanto, parece razoável supor que, no caso da assistência ao tuberculoso, a dimensão da transação incerteza é 'baixa'. Isto porque, dados os aspectos flutuação na demanda e nos preços dos insumos - nos quais a incerteza deve ser tomada (Ashton, 1998) -, pode-se considerar haver certa regularidade e previsibilidade no ambiente no qual a transação é realizada. Neste sentido, são mitigados também os impactos que tem a incerteza sobre a racionalidade, limitada, dos agentes, o que facilita o processo de tomada de decisão e, portanto, o planejamento da assistência ao tuberculoso. No que concerne, ainda, ao planejamento das ações, deve-se considerar a importância do SINAN, que dispõe livremente na internet informações sobre a epidemiologia da tuberculose.

Dado que os termos da transação são definidos em condições de informação incompleta (Bueno, 2004), no PNCT a racionalidade limitada deve surgir com maior ênfase na distribuição das competências entre as esferas federal, estadual e municipal, devido à possibilidade, na redação do contrato, de superposições e ambiguidades. Sabe-se, contudo, que a racionalidade limitada não remete, apenas, à questão da informação incompleta, mas tem a ver, sobretudo, com os limites cognitivos dos agentes envolvidos na transação. Isto porque, ainda que dispusessem de informação completa, não seriam, necessariamente, capazes de processá-las de modo a escolher a alternativa ótima, aquela cuja utilidade seria maximizada. Exclusive superposições e ambiguidades de competências, devido à informação incompleta, a questão da racionalidade limitada, no PNCT, não parece ser um problema para a realização da transação. A despeito da informação incompleta, que, por definição, faz parte de qualquer contrato, os termos da transação, no PNCT, podem ser considerados bem especificados. Por outro lado, na etapa que sucede a redação do contrato, surgem problemas de custos de transação ex post - no caso, avaliação, acompanhamento e monitoramento das ações previstas -, devido, principalmente, à possibilidade de comportamento oportunista por parte dos agentes envolvidos na relação contratual.

$\mathrm{Na}$ teoria dos custos de transação, os agentes econômicos são, por hipótese, oportunistas, “[...] no sentido de que se for de seu interesse, desistirão das obrigações pactuadas se não houver restrições suficientemente fortes" (Bueno 2004). 0 oportunismo costuma ser dividido em 'seleção adversa', ou oportunismo ex ante, e 'risco moral', ou oportunismo ex post. No primeiro caso, os 'agentes' possuem informações que, para o 'principal', são inobserváveis ou custosas de se obter antes de contratá-lo (hidden information). No segundo caso, as ações dos 'agentes' são escondidas do 'principal', ou são custosas de se observar durante o cumprimento do 'contrato' (hidden actions) (Arrow, 1985).

$\mathrm{Na}$ assistência ao tuberculoso, no Brasil, o problema do 'risco moral' deve surgir, com maior ênfase, no monitoramento das ações previstas no PNCT, ainda que disponibilizados os indicadores de acompanhamento - dado que esses indicadores monitoram os resultados, mas não as ações. Isto porque é difícil a implementação de mecanismos de monitoramento das ações dos agentes (gestores públicos de saúde, servidores públicos, médicos, enfermeiros e agentes de saúde) pelo 'principal' (a depender do contexto: Ministério da Saúde (MS), SES ou SMS). Dificuldade de monitoramento que é elevada por não se tratar o PNCT de um contrato explícito, no sentido de que não dispõe de um regime de incentivo bem definido, o que, segundo a TCT, aumenta a probabilidade de comportamento de tipo oportunista. Por sua vez, a 'seleção adversa', na assistência ao tuberculoso, deve se manifestar, com maior ênfase, na contratação de ativos humanos. Porém, devido à suposição de que o nível de habilidade requerido do ativo humano para executar a transação é, geralmente, baixo, parece razoável supor que a 'seleção adversa' não deve figurar como problema para a realização da transação. A baixa complexidade que envolve a execução 
da transação nos planos político, administrativo e operacional reduz, de certa forma, a posição privilegiada que tem, no momento da contratação, o agente sobre o principal. Desse modo, na assistência ao tuberculoso, admite-se ser 'alta' a probabilidade de ocorrência de comportamentos oportunistas por parte dos agentes envolvidos na transação. Fato devido, principalmente, ao risco moral.

\section{Resultados e Discussão}

Admitiu-se ser baixa a especificidade em ativos humanos na assistência ao tuberculoso. De forma a verificar a validade dessa afirmação, os entrevistados foram perguntados sobre aspectos ligados à contratação de pessoal qualificado e atuação dos profissionais; particularmente se são alocados exclusivamente na assistência ao tuberculoso. Os resultados das entrevistas sugerem haver grande dificuldade de contratação de pessoal - qualitativa e/ ou quantitativamente - para a realização da transação. As dificuldades de contratação parecem remeter ao regime de incentivos que rege as relações entre os agentes participantes da transação, principalmente os vínculos precários dos contratos, associados à baixa remuneração. Os contratos são ditos precários devido ao fato de que, usualmente, dada a escassez de concursos públicos para contratação permanente, os agentes são chamados a trabalhar por um período de tempo relativamente curto, normalmente dois anos - por exemplo, os contratados pelas Secretarias de Saúde através da UNESCO ou pelo Regime Especial de Direito Administrativo, o REDA. Um dos principais problemas apontados, derivados dos vínculos precários de contratação, foi a alta rotatividade do quadro de funcionários, dificultando a manutenção de um número mínimo adequado de profissionais para as demandas do serviço e gerando custos recorrentes com treinamento de pessoal.

Os profissionais que atuam na ponta da assistência ao tuberculoso (Médicos, Enfermeiros e Agentes de Saúde) não são alocados exclusivamente para lidar com o agravo. Dentre os motivos, relacionados pelos gestores, estão a qualificação profissional demandada, a escassez de profissionais e a descentralização do SUS. No que tange à qualificação, não é exigido dos profissionais formação especializada em tuberculose, porque o agravo, ou o nível de dificuldade para lidar com as dificuldades do problema, não demanda tal requisito. 0 quadro de escassez de profissionais, por sua vez, implica a necessidade de sua alocação para lidar com vários tipos de agravos, o que reforça a exigência de uma formação geral. Por último, a descentralização do SUS, no sentido de que não seria possível, no nível municipal, criar equipes para trabalhar exclusivamente com determinados agravos. Desse modo, a política consiste, de forma geral, em capacitar os profissionais em programas de atenção básica para que possam atuar, também, na assistência ao tuberculoso. Assim, os resultados sugerem ser baixa a especificidade em ativos humanos. Isto porque, para a realização da transação, não se faz necessária a contratação de pessoal especializado em tuberculose e, sendo assim, podem ser alocados em outras funções, sem perda de valor produtivo.

A 'incerteza' que permeia o ambiente de atuação dos agentes envolvidos na assistência ao tuberculoso foi discutida a partir de suposições apresentadas aos gestores, relativas à possibilidade de flutuação da demanda pelo serviço, introdução de novas tecnologias (medicamentos) e flutuação nos preços dos insumos (medicamentos). De forma geral, os respondentes consideraram haver certa regularidade e previsibilidade na procura pela assistência ao tuberculoso, permitindo o planejamento das ações pelos gestores públicos de saúde. As ações são, habitualmente, planejadas (i) a partir dos indicadores de acompanhamento do PNCT (indicadores de cobertura, processo e resultados) e (ii) da escolha de um dos dois, ou ambos, métodos de cálculo para incremento da oferta do serviço. 0 primeiro planeja a demanda futura estimando um acréscimo de $10 \%$ sobre o número de casos do último ano - mais utilizado em municípios que dispõem de uma melhor estrutura institucional e assistência à tuberculose consolidada - e o segundo a partir de um acréscimo de $1 \%$ sobre a população - porque se espera que tal percentual da população seja de sintomáticos e que $4 \%$ destes tenham tuberculose bacilífera. Este último método costuma ser usado em municípios cujas ações de combate à tuberculose ainda não estão consolidadas - são, em geral, municípios que apresentam um aparato institucional mais frágil. 
Segundo os respondentes, os métodos adotados permitem atender adequadamente as ações de planejamento do serviço.

Perguntou-se aos gestores quais seriam as prováveis consequências para a capacidade de prestação do serviço no caso de haver um elevado aumento na procura - em termos de infraestrutura disponível; dotação orçamentária; estoque de medicamentos; disponibilidade de pessoal qualificado; e contratação de pessoal qualificado. Sabe-se que o caráter endêmico da tuberculose faz mínima a possibilidade de ocorrência de um evento dessa natureza. Contudo, para fins de análise, a pergunta é válida na medida em que trata da incerteza relativa a flutuações da demanda no curto prazo. De forma geral, os gestores consideraram que um "choque" de demanda, caso houvesse, não figuraria como um problema para a capacidade de prestação do serviço, pois a estrutura existente permitiria absorvê-lo adequadamente. Os principais entraves relacionar-se-iam à disponibilidade e contratação de pessoal. Por outro lado, não haveria maiores problemas com a dotação orçamentária e o estoque de medicamentos. 0 primeiro, porque o programa de controle da tuberculose apresenta, usualmente, um custo não muito alto, bem como são previstos recursos para emergências epidemiológicas. 0 segundo, porque não tem havido problemas com o estoque e/ou distribuição de medicamentos - no caso extremo, o de desabastecimento, o MS poderia como já o fez, valer-se da importação de medicamentos.

Buscou-se investigar, também, quais os prováveis custos de mudança no caso da introdução de novas tecnologias (medicamentos). Observou-se que, para os PCTs estaduais e municipais, haveria, basicamente, na opinião dos gestores, custos de mudança derivados dos esforços necessários para capacitar os profissionais para lidar com a nova tecnologia. Admitiu-se, a partir da matriz de hipóteses, que não haveria possibilidade de oscilações na demanda em função da introdução de um novo medicamento, dado que o Governo Federal detém o monopólio da oferta. Segundo parte dos respondentes, a inovação na assistência ao tuberculoso é muito pouco provável, sobretudo em novos medicamentos, porque o mercado está excluído da transação. Assim, a pesquisa e desenvolvimento (P\&D) e inovação em medicamentos para a tuberculose pode ser considerada uma variável dependente da política do Governo Federal.

Não foi possível, porém, investigar, no âmbito dos PCTs, a questão relativa aos preços dos medicamentos: se são estáveis; com que frequência variam; qual o risco de desabastecimento derivado de uma grande elevação de preços. De forma geral, os gestores dos PCTs alegaram não dispor de informações para responder, porque a competência sobre medicamentos pertence à Assistência Farmacêutica.

Levando em conta o exposto, a incerteza que permeia o ambiente de atuação dos agentes no âmbito dos PCTs estaduais e municipais pode ser, então, considerada baixa - o que, de certa forma, reduz o impacto que tem o desconhecimento do futuro sobre o cálculo dos agentes. Neste ambiente, como dito, figura como fator redutor de incerteza o caráter endêmico da tuberculose, que permite o estabelecimento de um cálculo confiável de incremento da demanda.

Afirmou-se, por suposto, que, no PNCT, a racionalidade limitada deveria surgir com maior evidência na distribuição de competências entre as esferas federal, estadual e municipal; dada a possibilidade de superposições de competências e ambiguidades, derivadas da redação do contrato. No entanto, de forma geral, os gestores consideraram que os termos do contrato estão bem especificados, não apresentando superposições de competências e ambiguidades, o que, inclusive, facilita o cumprimento das ações e metas pactuadas. Neste sentido, pode-se admitir que a racionalidade limitada - no caso, a que remete à informação incompleta - não deve figurar como um problema relevante para a realização da transação.

Foi observado, também, nas entrevistas, o fluxo de informação nos PCTs pesquisados. Basicamente, foram mencionados desacertos no preenchimento dos livros de registro e dificuldades no envio de dados para o 'Sistema de Informação de Agravos de Notificação'. Porém, de forma geral, os entrevistados aprovam o SINAN, cuja importância para o planejamento das ações de controle e combate à tuberculose foi bastante ressaltada. Inobstante, afirmaram haver falhas no sistema, como, por exemplo: (i) erros no programa, como o derivado da transição do SINAN Windows para o SINAN NET, que, em 2007, prejudi- 
cou o acesso às informações na internet; (ii) erros na migração dos dados, que têm origem no fluxo de informações do agravo, do município (Secretarias Municipais de Saúde) até o Governo Federal (Ministério da Saúde); (iii) atraso de informações; e (iv) problemas na ponta da assistência ao tuberculoso, no município, derivado da digitação dos dados sobre o agravo, como, por exemplo, dupla contagem de pacientes e inserção de informações arbitrárias. Este último problema remete a situações como a seguinte: o SINAN demanda que determinadas informações sejam preenchidas para aceitar a inclusão dos dados do paciente no sistema, sem as quais não é possível fechar o cadastro. Como consequência, caso não tenham sido disponibilizadas pelo profissional de saúde que cuidou do paciente, o digitador pode optar por escolher, arbitrariamente, quais informações incluir, de modo a fechar o cadastro.

Com o objetivo de avaliar a possibilidade de ocorrência de comportamento oportunista por parte dos agentes envolvidos na transação, os gestores foram perguntados sobre: (i) a dificuldade de monitoramento das ações realizadas por Médicos, Enfermeiros e Agentes de Saúde, Gestores e Servidores Públicos; (ii) o regime de incentivos; (iii) e a aplicação de punições previstas no regime de direito administrativo, em caso de descumprimento das ações pactuadas no PNCT.

Argumentou-se, a partir da matriz de hipóteses, haver alta probabilidade de ocorrência de problemas de risco moral derivado da dificuldade de monitoramento das ações previstas no PNCT. De fato, pôde-se observar que o monitoramento das ações dos profissionais que atuam na assistência ao tuberculoso, seja de forma direta, seja indireta não é efetivamente realizado. Os gestores dos PCTs admitem as dificuldades de empreender monitoramento de ações, principalmente pela escassez de recursos humanos, estrutura disponível e regime de incentivos. 0 monitoramento das ações caberia aos PCTs municipais, enquanto que o das informações seria responsabilidade dos PCTs estaduais, a partir dos dados do SINAN. Os PCTs municipais, em tese, estariam em melhores condições que os estaduais para monitorar as ações realizadas na ponta do serviço - por Médicos, Enfermeiros e Agentes de Saúde. No entanto, seus gestores afirmam haver escassez de recursos humanos para tal atividade e, assim como nos PCTs estaduais, realizam, com maior frequência, o monitoramento de informações, a partir dos dados do livro de registro de sintomáticos respiratórios - que registra as informações sobre o paciente e o serviço.

Os achados da pesquisa sugerem que um dos principais problemas para o monitoramento das ações é o fraco regime de incentivos que rege a relação entre os agentes que participam da transação; o que, segundo a TCT, pode dar margem ao oportunismo. A contratação de médicos pode ser considerada um exemplo ilustrativo dessa situação. É sabida a dificuldade que têm os municípios, principalmente os mais pobres e afastados da capital, em atrair médicos para atuarem no Programa Saúde da Família (PSF) - no âmbito do qual são prestados os serviços de assistência ao tuberculoso. Essa situação, grosso modo, implica em deixar o poder de barganha para os médicos, em detrimento dos gestores municipais. Há, nesse sentido, um forte incentivo para o surgimento do risco moral, por parte do médico, na relação contratual. Isto porque é desinteressante, para o gestor, a opção pela aplicação de punições ao não cumprimento do contrato. Ou seja, no caso extremo, o de demissão do médico, a consequência seria penalizar a população usuária do serviço pela descontinuidade do tratamento. 0 médico demitido, muito provavelmente, dada a situação de escassez desse tipo de profissional, passaria, apenas, por uma situação de desemprego friccional (desemprego de curta duração).

Não existiria, na assistência ao tuberculoso, um regime de incentivos (que inclui punições) adequado para motivar os agentes ao cumprimento das ações contratadas - agentes que, na TCT, são, por definição, autointeressados e sujeitos a agir de forma oportunista. Para os respondentes, o maior incentivo dado aos agentes estaria nas atividades de capacitação profissional. No setor público, como sabido, não há incentivos de ordem financeira, como, por exemplo, um adicional que se paga quando um determinado agente cumpre uma meta estabelecida. Também foi verificado, junto aos gestores, que não são aplicadas punições no caso de descumprimento, pelos agentes, das ações contratadas. Assim, o fraco regime de incentivos que rege as relações entre os agentes 
parece potencializar, na assistência ao tuberculoso, o desalinhamento dos objetivos dos agentes em relação ao do principal, aumentando a probabilidade de ocorrência de comportamento oportunista - risco moral, principalmente.

Como sabido, as ações previstas no PNCT são pactuadas entre os entes federados. A pactuação, no entanto, faz parte do 'regime de direito administrativo' e, como tal, está sujeita às punições previstas na lei, em caso de descumprimento. Segundo os entrevistados, não são aplicadas, em geral, punições aos gestores de saúde, inclusive os arrolados na assistência ao tuberculoso. As punições previstas abrangeriam a interrupção de repasses de recursos federais para um dado município. O problema é que, em caso de aplicação da pena, o principal punido seria a população local usuária dos serviços públicos de saúde. Em dado regime de incentivo, a existência de uma punição que não pode ser aplicada - ou que pode, mas não é aplicada - pode induzir os agentes autointeressados ao comportamento oportunista. Nesse sentido, a não aplicação das sanções previstas no regime de direito administrativo pode gerar incentivo para que não sejam observadas, com o cuidado necessário, as ações pactuadas no PNCT. Desse modo, consoante com a TCT, pode-se admitir ser 'alta' a probabilidade de ocorrência de comportamentos oportunistas por parte dos agentes envolvidos na transação. Fato devido, principalmente, ao risco moral. Esse resultado apresenta-se consoante, também, com um estudo do World Bank (2007), o qual apontou haver, no âmbito do Sistema Único de Saúde, alta probabilidade de ocorrência de comportamentos dessa natureza.

A integração do serviço, a assistência ao tuberculoso, no âmbito do SUS, não é, obviamente, uma função da economia de custos de transação. Foi, dentre outros, motivada por fatores socioeconômicos. Como sabido, no Brasil, a tuberculose atinge, majoritariamente, a população mais pobre da sociedade, principalmente a que habita os bolsões de pobreza das grandes capitais - população que, também, dado o baixo nível de renda, não tem acesso à educação e saúde de qualidade. Assim, o perfil do cliente, o paciente, não atrai o interesse do capital privado por esse tipo de agravo, não somente no que tange à prestação do serviço, mas também à produção de medicamentos e inversão em P\&D. Esse contexto tornou imperiosa a integração da assistência ao tuberculoso no âmbito do SUS, dentre outros motivos, porque a solução de mercado não se afigurava factível.

O uso da teoria dos custos de transação para o estudo desse objeto não deve ser empregada para pensar qual seria, a partir das características da transação, a estrutura de governança mais adequada para economizar custos de transação (mercado, hierarquia ou formas híbridas). Isto porque a governança que rege a assistência ao tuberculoso é uma variável dependente da forma como está organizado o SUS. Neste sentido, os problemas derivados da forma como está organizada a assistência ao tuberculoso não são exclusivos deste agravo, mas, sim, comuns aos demais programas no âmbito do SUS. Assim, não é possível alterar a governança da assistência ao tuberculoso a partir dela mesma, porque o arranjo institucional atual é um dado do ambiente, dependente do SUS.

Desse modo, pode-se usar a TCT para pensar a melhoria da eficiência da transação a partir do arranjo existente. Ou seja, adequar a estrutura de governança em questão às características da transação específica, previstas no PNCT. Os resultados da pesquisa sugerem que o atual regime de incentivos - vigente no âmbito do SUS e secretarias estaduais e municipais de saúde - figura como um dos principais entraves para a busca do aumento da eficiência do arranjo institucional dos PCTs pesquisados. Isto porque, em parte, não logra êxito em alinhar os interesses do agente ao do principal, nos diferentes níveis do arranjo. Em outras palavras, os incentivos são fracos e as restrições não são suficientemente fortes para dissuadir o agente, autointeressado, do comportamento oportunista. O Quadro 2 apresenta uma síntese dos resultados do estudo.

Em parte, este estudo foi motivado pela constatação da enorme ampliação do espaço do conhecimento necessário para a prática da Saúde Coletiva, que não cabe na clássica, e importante, tríade Epidemiologia, Planejamento e Ciências Sociais. Várias disciplinas, espaços interdisciplinares e campos de saberes têm trazido aportes fundamentais para a compreensão da complexidade que envolve a saúde, sua prática e possibilidades de intervenção. Dentre elas, a Econo- 


\section{Quadro 2 - Síntese dos resultados do estudo.}

\begin{tabular}{|c|c|c|c|c|}
\hline $\begin{array}{l}\text { Características } \\
\text { da transação }\end{array}$ & Nivel & Tipo & Descrição & Resultados \\
\hline $\begin{array}{l}\text { Especificidade } \\
\text { de ativos }\end{array}$ & Baixa & Humanos & $\begin{array}{l}\text { Diretos: Médicos; } \\
\text { Enfermeiros; Agentes de } \\
\text { Saúde; } \\
\text { Indiretos: Gestores e } \\
\text { Servidores Públicos de } \\
\text { Saúde }\end{array}$ & $\begin{array}{l}\text { Dificuldade de contratação; baixa remuneração e caráter } \\
\text { precário dos contratos; alta rotatividade de profissionais; } \\
\text { dificuldades para a manutenção de um quadro } \\
\text { adequado à prestação do serviço; custos constantes } \\
\text { com treinamento; não são alocados exclusivamente } \\
\text { para a assistência ao tuberculoso; não são demandados } \\
\text { profissionais especializados em tuberculose; }\end{array}$ \\
\hline \multirow{2}{*}{ Incerteza } & \multirow{2}{*}{ Baixa } & \multirow{2}{*}{$\begin{array}{l}\text { Fundamental } \\
\text { (desconhecimento } \\
\text { do futuro) }\end{array}$} & Flutuação da demanda & $\begin{array}{l}\text { Regularidade e previsibilidade na procura pela } \\
\text { assistência ao tuberculoso; ambiente favorável ao } \\
\text { planejamento das ações pelos gestores públicos de } \\
\text { saúde; caráter endêmico da tuberculose funciona como } \\
\text { um fator redutor de incerteza; }\end{array}$ \\
\hline & & & $\begin{array}{l}\text { Introdução de novas } \\
\text { tecnologias }\end{array}$ & $\begin{array}{l}\text { Se introduzidas, figuraria como custo de mudança mais } \\
\text { relevante o custo com capacitação profissional; inovação } \\
\text { muito pouco provável, porque o mercado está excluído } \\
\text { da transação; introdução de novos medicamentos é uma } \\
\text { variável dependente do Governo Federal }\end{array}$ \\
\hline \multirow[b]{2}{*}{$\begin{array}{l}\text { Racionalidade } \\
\text { Limitada }\end{array}$} & \multirow[b]{2}{*}{ Baixa } & \multirow[b]{2}{*}{$\begin{array}{l}\text { Informação } \\
\text { incompleta }\end{array}$} & $\begin{array}{l}\text { Redação do contrato } \\
\text { (PNCT) }\end{array}$ & $\begin{array}{l}\text { Não há problemas com superposições de competências } \\
\text { e ambiguidades derivadas da redação do contrato, } \\
\text { considerado bem especificado; }\end{array}$ \\
\hline & & & SINAN & $\begin{array}{l}\text { Erros no programa; na inserção dos dados no sistema e } \\
\text { na migração; atraso das informações; dúvida quanto à } \\
\text { confiabilidade das informações disponibilizadas pelo } \\
\text { sistema; } \\
\text { Apesar de haver falhas no sistema, foi considerado } \\
\text { relevante para o planejamento das ações de controle e } \\
\text { combate à tuberculose; }\end{array}$ \\
\hline \multirow{3}{*}{ Oportunismo } & \multirow{3}{*}{ Alta } & \multirow{3}{*}{ Risco moral } & $\begin{array}{l}\text { Monitoramento das } \\
\text { ações }\end{array}$ & $\begin{array}{l}\text { Monitoramento das ações não é efetivamente realizado, } \\
\text { mas sim de informações (ou dos resultados); }\end{array}$ \\
\hline & & & Regime de incentivos & Inadequado; \\
\hline & & & $\begin{array}{l}\text { Punições em caso de } \\
\text { descumprimento das } \\
\text { ações pactuadas no PNCT }\end{array}$ & Não são aplicadas. \\
\hline
\end{tabular}

mia, cuja aplicação dos métodos e conceitos na área da investigação e estudos em saúde se restringia, até recentemente, a Financiamento, Avaliação Econômica, Análise de Custos, estudos de Farmacoeconomia e, mais raramente, estudos de Economia Política. Portanto, sem ênfase nos aspectos institucionais que envolvem a área da saúde.

Este trabalho parte do suposto que se deve reconhecer a importância fundamental da complexidade e variedade institucional na qual a problemática da saúde está imersa, pois os resultados das interven- ções em saúde são em grande parte dependentes das estruturas institucionais que regem as relações entre agentes. Como dito por Douglass North, Prêmio Nobel de Economia em 1993, as instituições são as "regras do jogo". A Teoria dos Custos de Transação, ao permitir lançar luz sobre aspectos institucionais e de condutas de agentes, é trazida, aqui, como contribuição à área da Saúde Coletiva no Brasil. 0 presente estudo consiste em uma primeira tentativa de aplicação deste referencial à prestação de serviços públicos de saúde no Brasil. A limitação dos achados 
empíricos, ora apresentados, apenas revela o estágio inicial desta discussão e que muito ainda deve ser feito para o desenvolvimento desta linha de investigação aplicada à Saúde Coletiva no Brasil.

\section{Referências}

ARIAS, X.; CABALLERO, G. Instituciones, costos de transacción y políticas públicas: un panorama. Revista de Economía Institucional, Bogotá, v. 5, n. 8, p. 117-146, 2003.

ARROW, K. The economics of agency. In: PRATT, J., ZECKHAUSER, J. (Ed.). Principals and agents: the structure of american business. Boston: Harvard Business School Press, 1985.

ASHTON, T. Contracting for health services in New Zealand: A transaction cost analysis. Social Science \& Medicine, Oxford/New York, v. 46, n. 3, p. 357-367, 1998.

ASHTON, T.; CUMMING, J.; MCLEAN, J. Contracting for health services in a public health system: the New Zealand experience. Health Policy, Amsterdam, v. 69, n. 1, p. 21-31, 2004.

BRASIL. Ministério da Saúde. Biblioteca Virtual em Saúde. Programa Nacional de Controle da Tuberculose. Brasília, DF, 2004. Disponível em: <http://bvsms.saude.gov.br/bvs/publicacoes/ ProgramaTB.pdf>. Acesso em: 3 fev. 2008.

BRASIL. Ministério da Saúde. Indicadores e Dados Básicos. Brasília, DF, 2006. Disponível em: $<$ http://www2.datasus.gov.br/DATASUS/index. php? area =0201>. Acesso em: 3 fev. 2008.

BRASIL. Ministério da Saúde. Sistema de Informações de Agravos de Notificação. Brasília, DF, [s.d.]. Disponível em: <http://dtrzoo4.saude. gov.br/sinanweb/index.php?name=Tnet $>$. Acesso em: 03 fev. 2008.

BROWN, T.; POTOSKI, M. Transaction costs and institutional explanations for government service production decisions. Journal of Public Administration Research and Theory. Oxford/New York, v. 13, n. 4, p. 441-468, 2003.

BUENO, N. Lógica da ação coletiva, instituições e crescimento econômico: uma resenha temática sobre a nova economia institucional. Economia, Brasília, v. 5, n. 2, p. 361-420, 2004.
COASE, R.H. The nature of the firm. Economica, London, v. 4, n. 16, p. 386-405, 1937.

DEQUECH, D. The new institutional economics and the theory of behaviour under uncertainty. Journal of Economic Behavior \& Organization, Amsterdam, v. 59, n. 1, p. 109-131, 2006.

\section{SECRETARIA MUNICIPAL DE SAÚDE DE} GOIÂNIA. Meningites. Boletim Epidemiológico, Goiânia, n. 2, 2007.

IBGE - INSTITUTO BRASILEIRO DE GEOGRAFIA E ESTATÍSTICA. Indicadores sociais. Rio de Janeiro, [s.d.]. Disponível em: <http://www.ibge.gov.br/ home/estatistica/populacao/condicaodevida/ indicadoresminimos/sinteseindicsociais2012/ default.shtm>. Acesso em: 3 fev. 2008.

MACHER, T.; RICHMAN, B. Transaction cost economics: an assessment of empirical research in the social sciences. Business and Politics, Berkeley, v. 10, v. 1, n. 1, p. 1-63, 2008.

PERES, U. Custos de transação e estrutura de governança no setor público. Revista Brasileira de Gestão de Negócios, São Paulo, v. 9, n. 24, p. 15-30, 2007.

PONDÉ, J. Coordenação e aprendizado: elementos para uma análise das inovações institucionais nas firmas e nos mercados. 1993. Dissertação (Mestrado em Economia) - Instituto de Economia, Universidade de Campinas, Campinas.

PREKER, A.; HARDING, A.; TRAVIS, P. "Make or buy" decisions in the production of health care goods and services: new insights from institutional economics and organizational theory. Bulletin of the World Health Organization, Geneva, v. 78, n. 6, p. 779-79o, 2000.

SANTOS-FILHO, E.; GOMES, Z. Estratégias de controle da tuberculose no Brasil: articulação e participação da sociedade civil. Revista de Saúde Pública, São Paulo, v. 1, n. 41, 2007.

SOUZA, W. et al.Tuberculose no Brasil: construção de um sistema de vigilância de base territorial. Revista de Saúde Pública, São Paulo, v. 39, n. 1, p. 82-89, 2005. 
UFBA - UNIVERSIDADE FEDERAL DA BAHIA.

Transaction costs analysis and tuberculosis in the decentralized Brazilian health system: relatório de pesquisa. Salvador: Instituto de Saúde Coletiva, 2007.

WILLIAMSON, O. Las instituciones económicas del capitalismo. Mexico: Fondo de Cultura Economica, 1989.

WILLIAMSON, O. Public and private bureaucracies: a transaction cost economics perspective. The Journal of Law, Economics, \& Organization, Oxford, New York, v. 15, n. 2, p. 306342, 1999.

WORLD BANK. Governance in Brazil's Unified Health System (SUS) raising the quality of public spending and resource management. Report. Washington, DC, 2007 (Report n. 36601-BR).

Recebido em: 03/01/2011

Reapresentado em: 29/09/20II

Aprovado em: 24/02/2012

98 Saúde Soc. São Paulo, v.22, n.I, p.85-98, 2013 